\title{
Mathematical modelling of the in-host dynamics of malaria and the effects of treatment
}

\author{
Zadoki Tabo*, Livingstone S. Luboobi, Joseph Ssebuliba \\ Department of Mathematics, Makerere University, Kampala, P. O. Box 7062, Uganda.
}

\begin{abstract}
Malaria research and mathematical models have mainly concentrated on malaria Plasmodium at the blood stage. This has left many questions concerning models of parasite dynamics in the liver and within the mosquito. These concerns are anticipated to keep scientists busy trying to understand the biology of the parasite for some more years to come. Thorough knowledge of parasite biology helps in designing appropriate drugs targeting particular stages of Plasmodium. To achieve this, there is need to study the transmission dynamics of malaria and the interaction between the infection in the liver, blood and mosquito using a mathematical model. In this study, a within-host mathematical model is proposed and considers the dynamics of P. falciparum malaria from the liver to the blood in the human host and then to the mosquito. Several techniques, including center manifold theory and sensitivity analysis are used to understand relevant features of the model dynamics like basic reproduction number, local and global stability of the disease-free equilibrium and conditions for existence of the endemic equilibrium. Results indicate that the infection rate of merozoites, the rate of sexual reproduction in gametocytes, burst size of both hepatocytes and erythrocytes are more sensitive parameters for the onset of the disease. However, a treatment strategy using highly effective drugs against such parameters can reduce on malaria progression and control the disease. Numerical simulations show that drugs with an efficacy above $90 \%$ boost healthy cells, reduce infected cells and clear parasites in human host. Therefore more needs to be done such as research in parasite biology and using highly effective drugs for treatment of malaria. (C)2017 All rights reserved.
\end{abstract}

Keywords: Malaria, malaria Plasmodium, sexual and asexual stages, stability and sensitivity analysis, treatment. 2010 MSC: 92D30, 34C60.

\section{Introduction}

Malaria is one of the old diseases that has remained a threat to human and animal populations [2, 19]. The disease continues to be one of the leading killer of humans worldwide especially in children under 5 years of age [70]. In 2012, 3.4 billions of people were at risk of malaria worldwide. Out of the estimated 207 million registered cases, 627,000 were confirmed dead [70]. Approximately 91\% of the confirmed death were from the African region and $85 \%$ were children under 5 years of age [70]. This makes malaria one of the most serious health challenges.

Malaria is caused by a single-celled genus Plasmodium transmitted by a female anopheles mosquito [26]. It can also be transmitted via blood transfusions, organ transplants, the shared use of

\footnotetext{
*Corresponding author

Email address: tabozac@gmail .com (Zadoki Tabo)
} 
needles or syringes contaminated with blood and from a mother to her fetus acquired during foetal development or at birth [10]. There are five parasite species of the genus Plasmodium (phylum Apicomplexa) that infect humans: Plasmodium vivax, Plasmodium ovale, Plasmodium malarie, Plasmodium falciparum and Plasmodium knowlesi $[18,60]$. Of the five species of malaria parasite, severe disease and deaths are overwhelmingly attributed to Plasmodium falciparum. It attacks both the young and old red blood cells. Next in the queue is Plasmodium vivax [11, 47, 53].

The symptoms of malaria appear in 10 to 14 days after a bite from an infectious mosquito. This coincides with the bursting of infected mature red blood cells called erythrocytes. These cells contain haemoglobin that carries oxygen to the body tissues [30]. Malaria typically produces a string of recurrent attacks, or paroxysms, each of which has three stages. The first stage is shaking chills, followed by fever, and then sweating. Along with chills, the person is likely to have headache, lassitude, abdominal discomfort, muscular pains, occasional nausea, vomiting, and diarrhea. After this stage, the body temperature rises and the skin feels hot and dry. The body temperature falls, sweating begins and the person feels tired, weak and is likely to fall asleep [69]. For severe and complicated malaria, symptoms such as splenomegaly, anemia, cerebral malaria, respiratory distress syndrome, acute renal failure, and in particular, convulsions may arise [47]. Unfortunately, in Africa, such symptoms are rarely associated with malaria and are frequently thought to be of supernatural or spiritual origin $[1,42,47]$.

The development of malaria Plasmodium is a complex process [25, 28, 29, 37, 39, 46] involving multiple stages which can be broken down into: sexual (sporogonic) and asexual stages which take place in the anopheles mosquitoes and in the humans respectively. The asexual stage involves exo-erythrocytics stage in the liver and erythrocytic stage in the blood. The parasites then exploit and feed on host cell resources like haemoglobin, causing acute haemolysis resulting into a condition known as blackwater fever. There is also indirect damage caused by release of toxic materials that trigger a chain of events like changes in capillary permeability, production of pharmacologically active peptides and anoxic anoxia $[25,41,43,51,54,67]$. This constitutes triggers the infected hepatocyte cells and the host counter-attacks by initiating defense programs aimed at eliminating the parasite or the infected cell. To survive, parasites must be able to successfully control their host hepatocyte [46]. At this stage, sporozoites undergo a process of remarkable growth, replication and asexual transformation into trophozoite form that produce tens of thousands of infectious red blood cells and haploid merozoites called schizonts.

The schizonts remain in a dormant state for the case of Plasmodium vivax and Plasmodium ovale and it can persist in the liver and cause relapses by invading the blood stream at later stages $[5,13]$. The liver stage is the most intractable and clinically inactive stage of the Plasmodium life cycle and it is experimentally inaccessible. The infected hepatocyte cells rupture when the parasites are fully developed and about 30,000 merozoites are released into the bloodstream [27]. In the bloodstream both the erythrocytes and the merozoites are in motion and their chance of meeting is high. The merozoites invade and infect erythrocytes. The invasion is a complex but extremely rapid process.

The parasites use the erythrocytes as sites of asexual replication by remodelling them. At this stage, they develop into defined ring trophozoite and schizont stages. They replicate to produce 16 to 32 daughter new merozoites during egress. The egress of Plasmodium falciparum involves a sudden increase in intracellular pressure together with biochemical changes that destabilize the infected cell cytoskeleton and these combine to promote an explosive event effectively dispersing the merozoites [29]. The free merozoites that are released are able to recognize, attack, invade and re-infect more new cells in the blood [17].

The process of infection is very rapid, short and highly efficient because the antigens on the surface of extracellular form of the parasite are particularly vulnerable to immune attack [30]. At the stage of infection, all the classic clinical symptoms of malaria paroxysms defined by intense chills, fever, and sweating arise. The frequency of paroxysms can vary depending on the speed of asexual replication and erythrocytes bursting. Plasmodium vivax, Plasmodium malariae, and Plasmodium ovale can all cause relapses; specifically, Plasmodium malariae can persist for decades before manifesting any symptoms. Plasmodium falciparum which is the main species of study can infect erythrocytes in any stage of erythrocyte 
development and can lead to an entire bloodstream being infected and far more frequent paroxysms than infections with other Plasmodium species [12].

The sexual forms of the parasite come about when some intra-erythrocytic blood stages develop and differentiate into male (microgamete) or female (macrogamete) gametocytes [59]. The gametocytes are either ingested by the mosquito into its gut or soon die and are removed by the human immune system. Within the mosquito, the male microgametes which are short lived penetrate rapidly and fertilise female macrogametes generating diploid zygotes [41]. Malaria parasites at this stage are hermaphrodites hence capable of self-fertalisation and cross-fertalisation in the presence of other clones [61]. The zygotes transform via an intermediate retort-form into ookinetes that surround a dense surface coat that provide protection against mosquito midgut digestive protease. Ingested asexual erythocytic-stage parasite and any gametocytes that have failed to transform into relatively resistant ookinete stage are also digested [57]. The ookinetes transform and develop into sedentary spherical oocystes [7, 63]. In each oocyst, DNA replication and protein synthesis is up regulated for asexual amplification. They grow in number and release subsequent sporozoites by budding [35]. The sporozoites penetrate and invade the mosquito's salivary gland secretory cavities [45].

During blood meal feeding, an anopheles mosquito injects a small proportion out of the hundreds of sporozoites into the human host during salivation discharge through the proboscis $[6,26]$. The inoculation of sporozoites into a new human host perpetuates the malaria life cycle. Once a mosquito is infected with the parasite, it stays that way for the rest of its life and is capable of infecting every susceptible host it bites [58]. This can lead to the disease onset, morbidity and death in the susceptible population. This would threaten Millennium Development Goals of reducing transmission of infection to other new healthy humans or mosquitoes, treating malaria to cure the infection, prevent progression to severe disease, prevent death and neurological deficit [70].

The discovery of drugs targeting either liver or sexual stage parasites faces even greater gaps in knowledge. The liver stage is clinically silent, intractable and the infected individuals remain asymptomatic in this stage. Moreover, at this stage, the parasite load is still low and the hepatocytes have in contrast to erythrocytes the capacity to present antigens to stimulate immune system, a factor ideal for treatment to control malaria progress [64].

In the case of asexual blood stage parasites, associated with clinical symptoms and the related mortality, WHO recommends use of combination therapy. These help to reduce infectious reservoir, prevent emergence and check on the rising threat of resistance to available drugs and to reduce the intolerable burden of malaria [69]. Some antimalarial therapies have been developed and classified as:

(i) Liver schizontocides;

include drugs for radical cure and prohylaxis such as 8-aminoquinolines and folate inhibitors. They suppress merozoites emerging from the liver.

(ii) Blood schizontocides;

act on the erythrocytes forms of the Plasmodium during asexual intra-erythrocytic stage e.g. Artimisinins, Quinolines-methanols (e.g. quinine and mefloquine), folate inhibitors which make easier the synthesis of folate (e.g. dapsone) and Atovaquone which affect mitochondrial function.

(iii) Causal prophylatic drugs;

used for chemoprophylaxis and they block link between exoerythrocytic stage and erythrocytic stage by killing and suppressing parasite densities when they emerge from the liver to prevent malaria attack. Such drugs include arteminin derivatives, chloroquine, lumefantrine, and doxycyline, and they are usually given in combination. These can also be given to individuals who intend to move to an area where malaria is endemic, one week before entering the area. 
(iv) Gametocytic inhibitors;

prevent transmission of parasite into mosquitoes and diminish the human reservoir of the disease. Such drugs include; pyrimethamine which has a potent sporontocidal action, primaquine which appears to act upon the gametocytes and also acts on the secondary exoerythrocytic liver schizonts that are responsible for relapse in symptoms of malaria that appear each other day due to Plasmodium vivax and Plasmodium ovale $[1,33,54,67]$.

At the blood stage, research has also successively identified several processes, such as hemoglobin degradation and heme detoxification, folate biosynthesis, and protein synthesis in the apicoplast, as effective targets for therapeutic intervention. This has lead to the development of different classes of drugs such as 4-aminoquinolines and the quinolinemethanols with their model of action concentrated inside the acidic digestive vacuole, where they are believed to bind $\beta$-hematin and interfere with heme detoxification. The falcipain inhibitors target cysteine proteases that participate in hemoglobin degradation in the digestive vacuole. Antibiotics such as azithromycin, doxycycline, and clindamycin act inside the chloroplast-like plastid organelle, where they inhibit protein translation, resulting in the death of the progeny of drug-treated parasites. Atovaquone and other selected compounds inhibit electron transport in the mitochondrion, whereas antifolates disrupt biosynthesis of folate in the cytosol [32]. If control programmes are to focus more on mortality and morbidity than on sheer incidence and prevalence, then this constitutes a challenge to develop malaria with-in host mathematical models that define the dynamics and the features of interaction between malaria parasites and the infected cells during the course of malaria parasites inside the body of the hosts.

Several mathematical malaria models have been formulated and studied at in-host levels with significant results towards evolution of malaria. The model by Anderson [3] which provides the basis for blood-stage Plasmodium falciparum has been extended in many directions to understand in-host behaviors of parasite. $[39,68]$, modified this model which lacked synchronicity to explain malaria dynamics like a per cycle multiplication rate and periodicity of malaria through the 3-compartments of red blood cells, infected red blood cells, and the merozoites. Many authors including: [4, 36, 38, 41, 47, 55] have interesting results in understanding the in-host modeling of immune response to malaria. In the work by Kamangira [44], modeled immune response and or drug intervention at the asexual blood stage of Plasmodium falciparum. Other scholars like Tumwiine and Forouzannia [24, 65] studied age compartmental models and used the system of age-structured partial equations integrated over age to a system of delay differential equations. While Nannyonga [52] considered the severe case of malaria and the results showed that malaria parasites can be absorbed in already infected red blood cells, causing faster rapture of cells and can lead anenia. However, all these scholars are limited to asexual blood stage of Plasmodium falciparum. In this study, we formulate a more detailed model to study the interaction of malaria parasite in sexual and asexual stages during its life cycle, i.e., we combine liver stage, blood stage and mosquito stage malaria into one mathematical model. Our goal is to study the interacting components in the entire complex malaria cycle through a mathematical model and carry out sensitivity analysis to determine parameters that drive the disease. We incorporate treatment at blood stage as control strategy on the most sensitive parameters and determine whether or not using our model, malaria can be greatly reduced in human host.

\section{Model formulation}

We develope a model that represents the dynamics of susceptible healthy hepatocytes $X(t)$, infected hepatocytes $Y(t)$, sporozoites in the infected liver $S(t)$, susceptible healthy erythrocytes $Z(t)$, infected erythrocytes $W(t)$, merozoites in the blood $M(t)$, gametocytes in the mosquito $G(t)$ and oocysts in the mosquito $\mathrm{C}(\mathrm{t})$ at time $\mathrm{t}$.

In constructing the model we assume the following in Subsection 2.1. 


\subsection{Assumptions}

(i) The susceptible hepatocytes are recruited at a constant rate and have a natural life expectancy.

(ii) The susceptible hepatocytes are infected at a rate proportional to the number of sporozoites inoculated into the liver from the mosquito.

(iii) The susceptible erythrocytes are recruited at a constant rate from the bone marrow and have a natural life expectancy.

(iv) The susceptible erythrocytes are infected at a rate proportional to their density in the blood.

(v) The released merozoites can either suffer from death naturally or successfully infect the erythrocytes.

(vi) Gametocytes in the human host are non-invasive and non-infective that their dynamics is ignored at human stage.

(vii) Susceptible mosquitoes repeatedly bite infected humans and they are capable of biting more susceptible humans hence initiating a new malaria cycle to new individuals in the community.

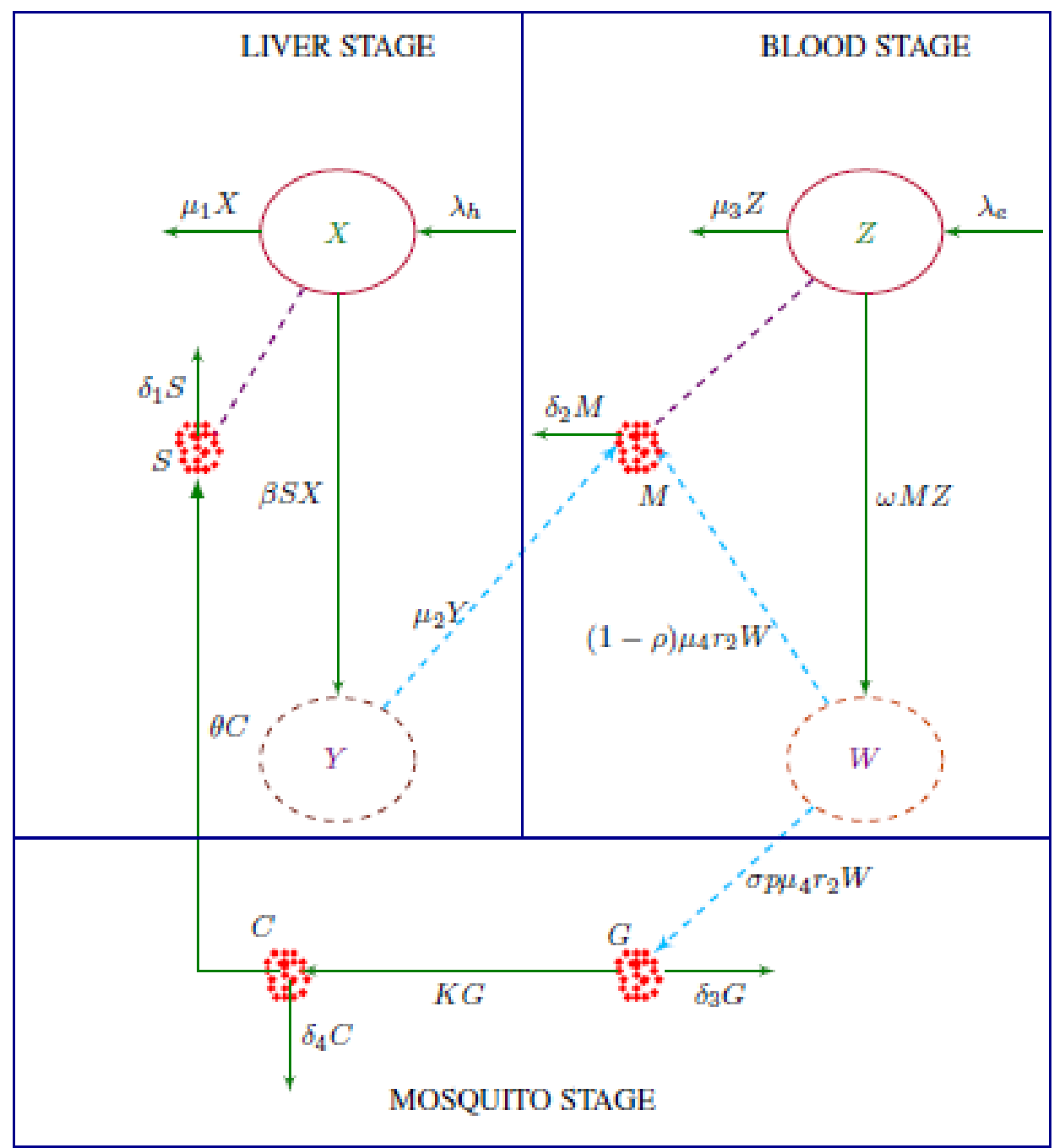

Figure 1: Model schemata for parasite dynamics. The dotted lines without arrows indicate cell-parasite interaction and dotted lines with arrows show the release of parasites from a bursting cell. The solid lines show progress from one class to another.

The transmission dynamics of malaria is summarised in the compartmental diagram in Figure 1. 


\subsection{Parameters}

The parameters used in the formulation of the model as indicated in Figure 1 are described as follows:

$\lambda_{h}$ : Recruitment rate of susceptible hepatocytes in the liver.

$\lambda_{e}$ : Recruitment rate of the susceptible erythrocytes from the bone marrow.

$\beta$ : Rate of infection of the hepatocyte by sporozoite.

$\omega$ : Rate of infection of the erythrocyte cells by merozoite.

$\mu_{1}$ : Natural death rate of hepatocyte cells.

$\mu_{2}$ : Death rate of infected hepatocyte cells.

$\mu_{3}$ : Natural death rate of erythrocyte cells.

$\mu_{4}$ : Death rate of infected erythrocyte cells.

$\delta_{1}$ : Per capita death rate of the sporozoite in the liver.

$\delta_{2}$ : Per capita death rate of merozoites.

$\delta_{3}$ : Per capita death rate of gametocytes.

$\delta_{4}$ : Per capita death rate of oocysts.

$r_{1}$ : The average number of merozoites produced per bursting infected hepatocyte cell.

$r_{2}$ : The average number (burst size) of parasites produced per bursting infected erythrocyte.

$\rho$ : Differentiation rate of the asexual form parasite that transform into gametocyte at the blood stage.

$\sigma:$ The rate at which the gametocytes are ingested from the human host into the mosquito during the blood meal.

$\phi:$ Rate at which the sporozoites are injected into the human host from the mosquito during a blood meal.

K: Rate of sexual reproduction in gametocytes to produce oocysts.

$\theta$ : Budding rate of infectious sporozoites from mature oocysts.

$r_{3}$ : A proportion of the mosquito resident sporozoites inoculated into the human host during blood feeding.

$\varepsilon_{1}$ : The drug efficacy or probability with which the blood schizontocides control the interaction and infection between erythrocytes and merozoites.

$\varepsilon_{2}$ : The drug efficacy or probability with which the blood schizontocides inhibit erythrocytic parasites including merozoites emerging from the liver. 


\subsection{The equations of the proposed model}

Putting the above description and assumptions of the dynamics of malaria together with treatment parameters $\varepsilon_{1}$ and $\varepsilon_{2}$, gives the following equations of the model:

$$
\begin{aligned}
\frac{\mathrm{dX}}{\mathrm{dt}} & =\lambda_{\mathrm{h}}-\mu_{1} \mathrm{X}-\beta X S, \\
\frac{\mathrm{dY}}{\mathrm{dt}} & =\beta X S-\mu_{2} Y, \\
\frac{\mathrm{dS}}{\mathrm{dt}} & =\phi r_{3} \theta \mathrm{C}-\delta_{1} S-\beta X S, \\
\frac{\mathrm{dZ}}{\mathrm{dt}} & =\lambda_{e}-\mu_{3} Z-\left(1-\varepsilon_{1}\right) \omega M Z, \\
\frac{\mathrm{dW}}{\mathrm{dt}} & =\left(1-\varepsilon_{1}\right) \omega M Z-\mu_{4} W, \\
\frac{\mathrm{dM}}{\mathrm{dt}} & =\left(1-\varepsilon_{2}\right) r_{1} \mu_{2} \mathrm{Y}-\delta_{2} M-\omega M Z+(1-\rho)\left(1-\varepsilon_{2}\right) r_{2} \mu_{4} W, \\
\frac{\mathrm{dG}}{\mathrm{dt}} & =\sigma \rho r_{2} \mu_{4} W-\delta_{3} G, \\
\frac{\mathrm{dC}}{\mathrm{dt}} & =\kappa G-\delta_{4} \mathrm{C}-\theta \mathrm{C} .
\end{aligned}
$$

\subsection{Positivity analysis}

For the malaria model to be biologically meaningful, it is important to show that the feasible solution set is positively-invariant set of the model.

Theorem 2.1. The region $\Omega \subset \mathbb{R}_{+}^{8}$ is positively invariant with respect to the system of equations (2.1) and a non-negative solution exists for all time $0<\mathrm{t}<\infty$.

Proof. If $\varepsilon_{1}=\varepsilon_{2}=0$ and $X(0)=X_{0}>0, Y(0)=Y_{0}>0, Z(0)=Z_{0}>0, S(0)=S_{0}>0, W(0)=W_{0}>$ $0, M(0)=M_{0}>0, G(0)=G_{0}>0, C(0)=C_{0}>0$ are the positive initial conditions, the solutions $X(t), Y(t), Z(t), S(t), W(t), M(t), G(t)$ and $C(t)$ of system (2.1) are all positive for all $t>0$ and if not, let there exist a time $t_{r}$ such that $X\left(t_{r}\right)=0, \quad X^{\prime}\left(t_{r}\right) \leqslant 0$, and $X(t)>0, Y(t)>0, Z(t)>0, S(t)>0, W(t)>$ $0, M(t)>0, G(t)>0$ and $C(t)>0$ for $0<t<t_{r}$. Suppose there exists a time $t_{m}$ such that $S\left(t_{m}\right)=0$, $S^{\prime}\left(t_{m}\right) \leqslant 0$, and $X(t)>0, Y(t)>0, Z(t)>0, S(t)>0, W(t)>0, M(t)>0, G(t)>0$ and $C(t)>0$ for $0<t<t_{m}$. This approach was also used by Magombedze [49]. It follows from equations (2.1) of the system that

$$
\begin{aligned}
\frac{d X\left(t_{r}\right)}{d t} & =\lambda_{h}-\mu_{1} X\left(t_{r}\right)-\beta X\left(t_{r}\right) S\left(t_{r}\right), \\
& =\lambda_{h}>0,
\end{aligned}
$$

which is a contradiction. For the latter case, equation (2.1) gives

$$
\begin{aligned}
\frac{\mathrm{d} S\left(t_{\mathrm{m}}\right)}{\mathrm{dt}} & =\phi r_{3} \theta C\left(t_{\mathrm{m}}\right)-\delta_{1} S\left(t_{\mathrm{m}}\right)-\beta X\left(\mathrm{t}_{\mathrm{m}}\right) \mathrm{S}\left(\mathrm{t}_{\mathrm{m}}\right), \\
& =\phi r_{3} \theta \mathrm{C}\left(\mathrm{t}_{\mathrm{m}}\right) \geqslant 0 .
\end{aligned}
$$

This is true if $C\left(t_{m}\right)=0$ or $C\left(t_{m}\right)>0$ and it follows that the solutions $X(t) \geqslant 0$ and $S(t) \geqslant 0$ are positive at any given time. Similarly, it can be shown that the variables $Y(t), Z(t), W(t), M(t), G(t)$ and $C(t)$ remain positive for all $t>0$. Thus the solutions of the system of equations (2.1) remain positive for all $t>0$. 


\subsection{Existence of equilibrium points}

The disease-free and the endemic equilibria are obtained by setting the rates of change or the left hand side of the equations of the system (2.1) to zero. Setting all the infected classes, that is, $\mathrm{Y}=\mathrm{S}=$ $\mathrm{W}=\mathrm{M}=\mathrm{G}=\mathrm{C}=0$, when $\varepsilon_{1}=\varepsilon_{2}=0$ and solving the resulting system at steady state, gives the disease-free equilibrium DFE as $E_{0}=\left(\frac{\lambda_{h}}{\mu_{1}}, 0,0, \frac{\lambda_{e}}{\mu_{3}}, 0,0,0,0\right)$. Expressing the endemic equilibrium point explicitly in only the parameters of the model is mathematically intractable. The components of the endemic equilibrium point $\left(X^{*}, Y^{*}, Z^{*}, S^{*}, W^{*}, M^{*}, G^{*}, C^{*}\right)$ are related through the following equations:

$$
\begin{aligned}
X^{*} & =\frac{\delta_{3} \lambda_{h}\left(\delta_{4}+\theta\right)\left(\delta_{1} \mu_{1}+\beta \lambda_{h}\right)\left(\mu_{3}+\omega M^{*}\right)}{\mu_{1} \mu_{3} \delta_{3}\left(\delta_{4}+\theta\right)\left(\delta_{1} \mu_{1}+\beta \lambda_{h}\right)+\left(\delta_{3}\left(\delta_{4}+\theta\right)\left(\delta_{1} \mu_{1}+\beta \lambda_{h}\right)+\beta \kappa \phi \theta \rho r_{2} r_{3} \lambda_{e}\right) M^{*}}, \\
Y^{*} & =\frac{\phi \theta \rho \mu_{1} \mu_{4} \kappa \omega r_{2} r_{3} \beta \lambda_{e} \lambda_{h} M^{*}}{\mu_{1} \mu_{2} \mu_{4} \delta_{3}\left(\delta_{4}+\theta\right)\left(\delta_{1} \mu_{1}+\beta \lambda_{h}\right)+\beta \kappa \rho \theta r_{2} r_{3} \mu_{1} \mu_{4} \omega \lambda_{e} M^{*}}, \\
S^{*} & =\frac{\phi \theta \rho \mu_{1} \mu_{4} \kappa \omega r_{2} r_{3} \lambda_{e} M^{*}}{\delta_{3} \mu_{4}\left(\delta_{4}+\theta\right)\left(\delta_{1} \mu_{1}+\beta \lambda_{h}\right)\left(\mu_{3}+\omega M^{*}\right)^{\prime}}, \\
Z^{*} & =\frac{\lambda_{e} \mu_{3} \mu_{4}}{\mu_{3} \mu_{4}\left(\mu_{3}+\omega M^{*}\right)^{\prime}}, \\
W^{*} & =\frac{\omega \lambda_{e} M^{*}}{\mu_{4}\left(\mu_{3}+\omega M^{*}\right)}, \\
M^{*} & =\frac{r_{1} \mu_{2} \mu_{3} Y^{*}+\mu_{4} \mu_{3}(1-\rho) r_{2} W^{*}}{\mu_{3} \delta_{2}+\omega \lambda_{h}-\omega \mu_{4} W^{*}}, \\
G^{*} & =\frac{\sigma \rho r_{2} \mu_{4} \omega \lambda_{e} M^{*}}{\delta_{3} \mu_{4}\left(\mu_{3}+\omega M^{*}\right)}, \\
C^{*} & =\frac{\sigma \rho r_{2} \mu_{4} \omega \lambda_{e} M^{*}}{\delta_{3} \mu_{4}\left(\mu_{3}+\omega M^{*}\right.} .
\end{aligned}
$$

\subsection{The basic reproduction number $\mathrm{R}_{0}$}

The within-host basic reproduction number $\left(R_{0}\right)$ of malaria Plasmodium is defined as the average number of secondary infected hepatocytes and infected erythrocytes caused by a single infective agent of malaria Plasmodium introduced in a population of purely susceptible hepatocytes and erythrocytes at disease-free equilibrium point. To analyze the stability of the disease free equilibrium point, we compute the basic reproduction number $R_{0}$ for the model by applying the next generation operator approach by Driessche and Watmough [21]. We obtain

$$
R_{0}=\frac{1}{2} \cdot\left(\frac{(1-\rho) r_{2} \omega \lambda_{e}}{\mu_{3} \delta_{2}+\omega \lambda_{e}}+\sqrt{\left(\frac{(1-\rho) r_{2} \omega \lambda_{e}}{\mu_{3} \delta_{2}+\omega \lambda_{e}}\right)^{2}+4\left(\frac{r_{1} \mu_{4} \omega \lambda_{e}}{\mu_{2}\left(\mu_{3} \delta_{2}+\omega \lambda_{e}\right)}\right)\left(\frac{r_{3} \theta \kappa \phi \rho \sigma r_{2} \beta \lambda_{h}}{\delta_{3}\left(\delta_{4}+\theta\right)\left(\mu_{1} \delta_{1}+\beta \lambda_{h}\right)}\right)}\right) .
$$

According to Driessche and Watmough [21], to develop a meaningful definition, the terms in $R_{0}$ can be interpreted as:

(i) $R_{1}=\frac{r_{1} \mu_{4} \omega \lambda_{e}}{\mu_{2}\left(\mu_{3} \delta_{2}+\omega \lambda_{e}\right)}$ is the expected number of secondary infection of erythrocytes in the blood caused by merozoites released from a single bursting infected hepatocyte cell in the liver. This shows that there exists a critical value $r_{1}^{c}=\frac{\mu_{2}\left(\delta_{2} \mu_{3}+\omega \lambda_{e}\right)}{\mu_{4} \omega \lambda_{e}}$ of merozoites from the liver above which the infection will progress to the blood stage and vice versa.

(ii) $R_{2}=\frac{(1-\rho) r_{2} \omega \lambda_{e}}{\mu_{3} \delta_{2}+\omega \lambda_{e}}$ is the expected number of newly secondary infections due to re-infection caused by new daughter merozoites released from a single bursting infected erythrocyte. $r_{2}^{c}=\frac{\mu_{4} r_{1}^{c}}{\mu_{2}}$ is the 
critical value of daughter merozoites from the blood above which the infection will persist in the blood and vice versa.

(iii) $R_{3}=\frac{r_{3} \theta \kappa \phi \rho \sigma r_{2} \beta \lambda_{h}}{\delta_{3}\left(\delta_{4}+\theta\right)\left(\mu_{1} \delta_{1}+\beta \lambda_{h}\right)}$ is the expected number of secondary infections of the hepatocytes of the liver caused by sporozoites. The terms in $R_{3}$ show that the number of new infections in the liver depend on the number of gametocytes originally from the blood stage and the number of sporozoites in the mosquito, where $\frac{\delta_{3}\left(\delta_{4}+\theta\right)}{r_{2} \rho \sigma}$ is the critical number of gametocytes introduced into the mosquito from the blood and $r_{3}^{c}=\frac{\left(\delta_{1} \mu_{1}+\beta \lambda_{h}\right)}{\theta \kappa \phi \beta \lambda_{h}}$ is apportion of the mosquito's resident sporozoite introduced into the liver above which the infection will persist in the liver.

\subsection{Local stability of DFE point $\mathrm{E}_{0}$}

The local stability of the disease-free equilibrium points can be established by showing that all the eigenvalues of the Jacobian matrix of the linearized system [1] evaluated $E_{0}$ are negative and is based on the following theorem:

Theorem 2.2. The disease free equilibrium $\mathrm{E}_{0}$ of the system (2.1) is locally asymptotically stable in $\Omega$ if $\mathrm{R}_{0}<1$ and unstable, if $\mathrm{R}_{0}>1$, where $\mathrm{R}_{0}$ is the reproduction number.

Proof. The Jacobian matrix J at the disease-free equilibrium point is obtained as

$J\left(E_{0}\right)=\left(\begin{array}{cccccccc}-\mu_{1} & 0 & -\beta \frac{\lambda_{h}}{\mu_{1}} & 0 & 0 & 0 & 0 & 0 \\ 0 & -\mu_{2} & \beta \frac{\lambda_{h}}{\mu_{1}} & 0 & 0 & 0 & 0 & 0 \\ 0 & 0 & -\delta_{1}-\beta \frac{\lambda_{h}}{\mu_{1}} & 0 & 0 & 0 & 0 & \phi r_{3} \theta \\ 0 & 0 & 0 & -\mu_{3} & 0 & -\omega \frac{\lambda_{e}}{\mu_{3}} & 0 & 0 \\ 0 & 0 & 0 & 0 & -\mu_{4} & \omega \frac{\lambda e}{\mu_{3}} & 0 & 0 \\ 0 & r_{1} \mu_{2} & 0 & 0 & (1-\rho) r_{2} \mu_{4} & -\left(\delta_{2}+\omega \frac{\lambda_{e}}{\mu_{3}}\right) & 0 & 0 \\ 0 & 0 & 0 & 0 & \sigma \rho r_{2} \mu_{4} & 0 & -\delta_{3} & 0 \\ 0 & 0 & 0 & 0 & 0 & 0 & \kappa & -\left(\delta_{4}+\theta\right)\end{array}\right)$.

By inspection, two of the eigenvalues are $-\mu_{1}$ and $-\mu_{3}$. The remaining six eigenvalues are obtained by solving the characteristic polynomial of a $6 \times 6$ block matrix below

$$
\left|\begin{array}{cccccc}
-\mu_{2} & \beta \frac{\lambda_{h}}{\mu_{1}} & 0 & 0 & 0 & 0 \\
0 & -\delta_{1}-\beta \frac{\lambda_{h}}{\mu_{1}} & 0 & 0 & 0 & \phi r_{3} \theta \\
0 & 0 & 0 & \omega \frac{\lambda_{e}}{\mu_{3}} & 0 & 0 \\
r_{1} \mu_{2} & 0 & (1-\rho) r_{2} \mu_{4} & -\left(\delta_{2}+\omega \frac{\lambda_{e}}{\mu_{3}}\right) & 0 & 0 \\
0 & 0 & \sigma \rho r_{2} \mu_{4} & 0 & -\delta_{3} & 0 \\
0 & 0 & 0 & 0 & \kappa & -\left(\delta_{4}+\theta\right)
\end{array}\right|=0 .
$$

The characteristic polynomial is given implicitly as;

$$
y^{6}+a_{1} y^{5}+a_{2} y^{4}+a_{3} y^{3}+a_{4} y^{2}+a_{5} y+a_{6}=0,
$$


where,

$$
\left\{\begin{aligned}
a_{1} & =\mu_{3}+\mu_{4}-\delta_{1}+\delta_{2}+\delta_{3}-\delta_{4}-\theta+\frac{\omega \lambda_{e}}{\mu_{3}}, \\
a_{2} & =\mu_{2} \delta_{3}+\left(1-\delta_{1}-\delta_{4}-\theta\right)\left(\delta_{3}+\mu_{3}\right)-\left(\frac{\omega \lambda_{e}}{\mu_{3}}-\mu_{4}-\delta_{1}\right)\left(\delta_{4}+\theta\right)-\delta_{1}, \\
a_{3}= & \left(\delta_{1}+\delta_{4}+\theta\right)\left(\delta_{3}+\mu_{3}\right)\left(\frac{\omega \lambda_{e}}{\mu_{3}}-\mu_{4}\right)-\left(1+\delta_{1}+\delta_{4}+\theta\right) \mu_{2} \delta_{3} \\
a_{4}= & \left(\left(\delta_{1}+\delta_{4}+\theta\right)\left(\delta_{3}+\mu_{2}\right)+\delta_{1}\left(\delta_{4}+\theta\right)-\delta_{3} \mu_{2}\right)\left(\frac{\omega \lambda_{e}(1-\rho) r_{2} \mu_{4}}{\mu_{3}}+\mu_{4}\left(\delta_{2}+\omega \frac{\lambda_{e}}{\mu_{3}}\right)\right)+ \\
& \left(\left(\delta_{1}+\delta_{4}+\theta\right) \mu_{2} \delta_{3}-\delta_{1}\left(\delta_{4}+\theta\right)\left(\delta_{3}+\mu_{2}\right)\right)\left(\mu_{4}+\left(\delta_{2}+\omega \frac{\lambda_{e}}{\mu_{3}}\right)\right)+\delta_{1}\left(\delta_{4}+\theta\right) \mu_{3}-2 \delta_{1}, \\
a_{5}= & \delta_{1}^{2} \mu_{3}\left(\delta_{4}+\theta\right)\left(\mu_{4}+\delta_{2}+\frac{\omega \lambda_{e}}{\mu_{3}}\right)+\left(\left(\delta_{1}+\delta_{2}+\theta\right) \delta_{3} \mu_{2}+\delta_{1}\left(\delta_{4}+\theta\right)\left(\mu_{2}-\delta_{3}\right)\right) \frac{\omega \lambda_{e}(1-\rho) r_{2} \mu_{4}}{\mu_{3}} \\
& -\left(\delta_{1}\left(\delta_{4}+\theta\right)\left(\mu_{2}+\delta_{3}\right)-\left(2 \delta_{1}+\delta_{4}+\theta\right) \mu_{3} \delta_{3}\right)-\mu_{4}\left(\delta_{2}+\omega \frac{\lambda_{e}}{\mu_{3}}\right), \\
a_{6}= & \delta_{1}^{2}\left(\delta_{4}+\theta\right) \mu_{4} \omega \lambda_{e}+\delta_{1} \delta_{3} \mu_{2}\left(\delta_{4}+\theta\right) \frac{\omega \lambda_{e}(1-\rho) r_{2} \mu_{4}}{\mu_{3}} .
\end{aligned}\right.
$$

By using the Routh-Hurwitz stability criterion, the steady state associated with the characteristic polynomial is stable, if and only if the determinants of all the Hurwitz matrices are positive, that is, $\operatorname{Det}\left(H_{j}\right)>0, j=1,2, \cdots, k$. The Hurwitz matrices of the characteristic polynomial are:

$$
\begin{gathered}
H_{1}=\left(a_{1}\right), \quad H_{2}=\left(\begin{array}{cc}
a_{1} & 1 \\
a_{3} & a_{2}
\end{array}\right), \quad H_{3}=\left(\begin{array}{ccc}
a_{1} & 1 & 0 \\
a_{3} & a_{2} & a_{1} \\
a_{5} & a_{4} & a_{3}
\end{array}\right), \quad H_{4}=\left(\begin{array}{cccc}
a_{1} & 1 & 0 & 0 \\
a_{3} & a_{2} & a_{1} & 1 \\
a_{5} & a_{4} & a_{3} & a_{2} \\
0 & a_{6} & a_{5} & a_{4}
\end{array}\right), \\
H_{5}=\left(\begin{array}{ccccc}
a_{1} & 1 & 0 & 0 & 0 \\
a_{3} & a_{2} & a_{1} & 1 & 0 \\
a_{5} & a_{4} & a_{3} & a_{2} & a_{1} \\
0 & a_{6} & a_{5} & a_{4} & a_{3} \\
0 & 0 & 0 & a_{6} & a_{5}
\end{array}\right), \quad H_{6}=\left(\begin{array}{cccccc}
a_{1} & 1 & 0 & 0 & 0 & 0 \\
a_{3} & a_{2} & a_{1} & 1 & 0 & 0 \\
a_{5} & a_{4} & a_{3} & a_{2} & 0 & 0 \\
0 & a_{6} & a_{5} & a_{4} & a_{3} & 0 \\
0 & 0 & 0 & a_{6} & a_{5} & 0 \\
0 & 0 & 0 & 0 & 0 & a_{6}
\end{array}\right) .
\end{gathered}
$$

The Routh-Hurwitz criterion applied to the characteristic polynomial requires that $a_{i}>0$ for all $i=1,2, \cdots, 6$ and

$$
\begin{aligned}
a_{1} a_{2} a_{3} a_{4} a_{5}+a_{6} a_{3}^{3}+a_{1}^{2} a_{3} a_{4} a_{6}+a_{1}^{2} a_{2} a_{5} a_{6}+2 a_{1} a_{4} a_{5}^{2}+a_{2} a_{3} a_{5}^{2} \\
-a_{3}^{2} a_{4} a_{5}-2 a_{1} a_{3} a_{5} a_{6}-a_{1} a_{2} a_{6} a_{3}^{2}-a_{1}^{2} a_{4}^{2} a_{5}-a_{1} a_{2}^{2} a_{5}^{2}-a_{5}^{3}>0 .
\end{aligned}
$$

Numerically using model parameter values, gives $a_{1}=5.700, a_{2}=33.0961, a_{3}=18.8028, a_{4}=$ 3402.3, $a_{5}=5372.2$ and $a_{6}=244.0913$, affirms that $a_{i}>0$ for all $i=1,2, \cdots, 6$. Therefore the condition above is greater than zero if:

$$
\begin{aligned}
a_{1} a_{2} a_{3} a_{4} a_{5}+a_{6} a_{3}^{3}+a_{1}^{2} a_{3} a_{4} a_{6}+a_{1}^{2} a_{2} a_{5} a_{6}+2 a_{1} a_{4} a_{5}^{2}+a_{2} a_{3} a_{5}^{2}> & a_{3}^{2} a_{4} a_{5}+2 a_{1} a_{3} a_{5} a_{6} \\
& +a_{1} a_{2} a_{6} a_{3}^{2}+a_{1}^{2} a_{4}^{2} a_{5}+a_{1} a_{2}^{2} a_{5}^{2}+a_{5}^{3} .
\end{aligned}
$$

This condition implies that all the eigenvalues are negative and $\mathrm{R}_{0}<1$, hence the disease-free equilibrium point is locally asymptotically stable. 


\subsection{Global stability of DFE point $\mathrm{E}_{0}$}

According to Castillo-Chavez [14], two conditions guarantee global asymptotic stability of the diseasefree state. Consider the transformation of system (2.1) into

$$
\left\{\begin{array}{l}
\frac{d M}{d t}=F(M, N), \\
\frac{d N}{d t}=G(M, N), \quad G(M, N)=0,
\end{array}\right.
$$

where $M=(X, Z), M \in \mathbb{R}^{2}$ denotes uninfected populations of the cells and $N=(Y, S, W, M, G, C)$, $N \in \mathbb{R}^{6}$ denotes the infectious classes. $E_{0}=\left(M^{*}, 0\right)$ represents the disease-free equilibrium of system (2.2). The following two conditions guarantee global asymptotic stability.

(i) for $\frac{d M}{d t}=F(M, 0), \quad M^{*}$ is globally asymptotically stable.

(ii) $\mathrm{G}(M, N)=D_{z} G\left(M^{*}, 0\right) N-\widehat{G}(M, N), \widehat{G}(M, N) \geqslant 0$, for $(M, N) \in \Omega$,

where $D_{z} G\left(M^{*}, 0\right)$ is an M-matrix (the off diagonal elements are nonnegative) and is also the Jacobian of $G(M, N)$ taken in $(Y, S, W, M, G, C)$ and evaluated at $M^{*}=\left(\frac{\lambda_{h}}{\mu_{1}}, 0,0, \frac{\lambda_{e}}{\mu_{3}}, 0,0,0,0\right)$. If system (2.2) satisfies the conditions (i) and (ii) above, then according to Castillo-Chavez [14] the following theorem holds.

Theorem 2.3. The equilibrium point $\mathrm{E}_{0}=\left(\mathrm{M}^{*}, 0\right)$ of system (2.1) is globally asymptotically stable if $\mathrm{R}_{0} \leqslant 1$ given that conditions (i) and (ii) are satisfied.

Proof. From (2.2), the two functions $\mathrm{F}(\mathrm{M}, \mathrm{N})$ and $\mathrm{G}(\mathrm{M}, \mathrm{N})$ are given by

$$
\begin{aligned}
& F(M, N)=\left(\begin{array}{c}
\lambda_{h}-\mu_{1} X-\beta X S \\
\lambda_{e}-\mu_{3} Z-\omega M Z .
\end{array}\right) \\
& G(M, N)=\left(\begin{array}{c}
\beta X S-\mu_{2} Y \\
\phi r_{3} \theta C-\delta_{1} S-\beta X S \\
\omega M Z-\mu_{4} W \\
r_{1} \mu_{2} Y-\delta_{2} M-\omega M Z+(1-\rho) r_{2} \mu_{4} W \\
\sigma \rho r_{2} \mu_{4} W-\delta_{3} G \\
\kappa G-\delta_{4} C-\theta C .
\end{array}\right) .
\end{aligned}
$$

We then consider the reduced system $\frac{d M}{d t}=F(M, 0)$ from condition (i),

$$
F(M, 0)=\left\{\begin{array}{l}
\lambda_{h}-\mu_{1} X, \\
\lambda_{e}-\mu_{3} Z
\end{array}\right.
$$

$M^{*}=\left(\frac{\lambda_{h}}{\mu_{1}}, \frac{\lambda_{e}}{\mu_{3}}\right)$ is globally asymptotically stable for the reduced system (2.3). The first equation of system (2.3) gives $X(t)=\frac{\lambda_{h}}{\mu_{1}}+\left(X(0)-\frac{\lambda_{h}}{\mu_{1}}\right) e^{-\mu_{1} t}$, which approaches $\frac{\lambda_{h}}{\mu_{1}}$ as $t \rightarrow \infty$. Similarly from the second equation of system (2.3), $Z(t)=\frac{\lambda_{e}}{\mu_{3}}+\left(Z(0)-\frac{\lambda_{e}}{\mu_{3}}\right) e^{-\mu_{3} t}$, which approaches $\frac{\lambda_{e}}{\mu_{3}}$ as $t \rightarrow \infty$. Note that this asymptomatic dynamics is independent of the initial conditions in $\Omega$. Therefore, convergence of the solutions of system (2.3) is global in $\Omega$. We compute for $G(M, N)=D_{z} G\left(M^{*}, 0\right) N-\widehat{G}(M, N)$ and show that $\widehat{\mathrm{G}}(\mathrm{M}, \mathrm{N}) \geqslant 0$. 


$$
\begin{gathered}
\mathrm{D}_{z} \mathrm{G}\left(M^{*}, 0\right)=\left(\begin{array}{cccccc}
-\mu_{2} & \beta X_{0} & 0 & 0 & 0 & 0 \\
0 & -\delta_{1}-\beta X_{0} & 0 & 0 & 0 & \phi r_{3} \theta \\
0 & 0 & -\mu_{4} & \omega Z_{0} & 0 & 0 \\
r_{1} \mu_{2} & 0 & (1-\rho) r_{2} \mu_{4} & -\delta_{2}-\omega Z_{0} & 0 & 0 \\
0 & 0 & \sigma \rho r_{2} \mu_{4} & 0 & -\delta_{3} & 0 \\
0 & 0 & 0 & 0 & \kappa & -\delta_{4}-\theta
\end{array}\right), \\
\hat{G}(M, N)=\left(\begin{array}{cccccc}
0 & 0 & 0 & 0 & 0 & 0
\end{array}\right)^{\top}=0 .
\end{gathered}
$$

Thus all terms of $\hat{G}(M, N)$ are zeros which are non-negative, that is to say $\hat{G}(M, N)=0$ hence Theorem 2.3 holds.

\section{Sensitivity analysis of $R_{0}$}

The sensitivity analysis is performed to quantify the effects of change in the model parameters of $R_{0}$ and to determine the relative importance of different parameters. It helps to understand which malaria Plasmodium interferes with treatment. The normalized forward sensitivity index approach by Kungaro [48] is used to quantify the effect of change in the model parameters. Due to the limited availability of literature and data especially at the liver and mosquito stages, the qualitative predictions of our model is dependent on estimating some of the parameter values.

\begin{tabular}{|c|c|c|c|}
\hline Parameter & Range of values & References & indices \\
\hline$\lambda_{h}$ & $3 \times 10^{8}$ cells $/ \mathrm{ml} /$ day & Mota et al. (2001) [51] & 0.0600 \\
\hline$\lambda_{e}$ & $2.5 \times 10^{8}$ cells $/ \mathrm{ml} /$ day & Austin et al. (1998) [4] & 0.7931 \\
\hline$\beta$ & $4.0 \times 10^{-9} /$ sporozoite/day & Estimated & 0.0600 \\
\hline$\omega$ & $2.0 \times 10^{-10} /$ merozoite/day & Dondorp et al. (2000) [19] & 0.7931 \\
\hline$\mu_{1}$ & $0.0022-0.0067 /$ day & Mota et al. (2001) [51] & -0.0600 \\
\hline$\mu_{2}$ & $2 /$ day & Estimated & -0.0824 \\
\hline$\mu_{3}$ & $0.0083 /$ day & Anderson et al. (1989) $[2,3]$ & 0.7107 \\
\hline$\mu_{4}$ & $0.24 /$ day & Chiyaka et al. (2010) [16] & 0.0824 \\
\hline$\delta_{1}$ & 48/day & Estimated & -0.0600 \\
\hline$\delta_{2}$ & 48/day & Austin et al. (1998) [4] & -0.7931 \\
\hline$\delta_{3}$ & $0.06 /$ day & Hellriegel (1992) [36] & -0.0824 \\
\hline$\delta_{4}$ & $0.071-0.143 /$ day & Howells and Davies,(1971) [40] & -0.0028 \\
\hline$r_{1}$ & 16/hepatocyte/day & Estimated & 0.0824 \\
\hline$r_{2}$ & 16/erythrocyte/day & Chiyaka et al. (2010) [16] & 1 \\
\hline$r_{3}$ & 10-100/mosquito/bite & Hall and Fauci, (2009) [34] & 0.0824 \\
\hline$\sigma$ & $0.833 /$ mosquito/bite & Esteva et al. (2009) [22] & 0.0824 \\
\hline k & $0.01-0.05 /$ gametocyte/day & Gouagna et al. (2004)[31] & 0.4240 \\
\hline$\theta$ & $2 /$ day & Estimated & 0.0824 \\
\hline$\rho$ & 0.5 dimensionless & Drodorp et al. (2000) [19] & -0.7527 \\
\hline$\phi$ & $0.181 /$ mosquito/bite & Esteva et al. (2009) [22] & 0.0824 \\
\hline
\end{tabular}

Table 1: Parameter values of model, their source and indices:

It is observed that $r_{2}$ is the most sensitive parameter. This is because the released daughter merozoites re-infect the erythrocytes in the blood and contribute to malaria progression. Other positively 
sensitive parameters to the progression of the disease are: $\omega, \lambda_{e}, r_{1}, r_{3}, \sigma, \phi, \kappa, \mu_{4}, \lambda_{h}, \beta, \theta$. The sensitivity indices of the remaining model parameters to $R_{0}$ are negatives and they have no effect on the onset of the infection. In order to have $1 \%$ decrease in the value of $R_{0}$, it is necessary to decrease the value of $r_{2}, \omega, \lambda_{e}, r_{1}, r_{3}, \sigma, \phi, \kappa, \mu_{4}, \lambda_{h}, \beta$ or $\theta$ by $1 \%, 0.7931 \%, 0.7931 \%, 0.0824 \%, 0.0824 \%, 0.0824 \%, 0.0824 \%$, $0.0824 \%, 0.0824 \%, 0.0600 \%, 0.0600 \%$, or $0.0028 \%$ respectively. While in order to have $1 \%$ decrease in the value of $R_{0}$, it is necessary to increase the value of $\delta_{4}, \mu_{1}, \delta_{1}, \mu_{2}, \delta_{3}, \mu_{3}, \rho$ and $\delta_{2}$ by $0.0028 \%, 0.0600 \%$, $0.0600 \%, 0.0824 \%, 0.0824 \%, 0.7107 \%, 0.7527 \%$, and $0.7931 \%$ respectively. This means that increasing parameters with positive indices enhances malaria progression while increasing parameters with negative indices results in low values of $R_{0}$ and reduces malaria propagation. This suggests that suppressing the numbers of parasite populations $r_{2}$ and $r_{1}$ as well as reducing the infection rate $\omega$, helps to reduce the value of $R_{0}$.

\section{Treatment effects}

The blood schizontocides inhibit the interaction between erythrocytes and merozoites, they block the link between exo-erythrocytic and erythrocytic stage hence suppressing merozoites from the liver and those within the blood stream. The infection rate of erythrocytes reduces to $\left(1-\varepsilon_{1}\right) \omega$ and the merozoites from the liver are suppressed at a rate $\left(1-\varepsilon_{2}\right) r_{1}$, while the burst size of infected erythrocytes becomes $\left(1-\varepsilon_{2}\right) r_{2}$. Using the next generation method, the treatment reproduction number $R_{\varepsilon}$ of system (2.1) in the presence of blood antimalarials is obtained as

$$
\begin{aligned}
R_{\varepsilon}= & \frac{1}{2} \cdot \frac{(1-\rho)\left(1-\varepsilon_{1}\right)\left(1-\varepsilon_{2}\right) r_{2} \omega \lambda_{e}}{\mu_{3} \delta_{2}+\left(1-\varepsilon_{1}\right) \omega \lambda_{e}} \\
& +\frac{1}{2} \cdot \sqrt{\left(\frac{(1-\rho)\left(1-\varepsilon_{1}\right)\left(1-\varepsilon_{2}\right) r_{2} \omega \lambda_{e}}{\mu_{3} \delta_{2}+\left(1-\varepsilon_{1}\right) \omega \lambda_{e}}\right)^{2}+4\left(\frac{\left(1-\varepsilon_{1}\right)\left(1-\varepsilon_{2}\right) r_{1} \mu_{4} \omega \lambda_{e}}{\mu_{2}\left(\mu_{3} \delta_{2}+\left(1-\varepsilon_{1}\right) \omega \lambda_{e}\right)}\right)\left(\frac{r_{3} \theta \kappa \phi \rho \sigma\left(1-\varepsilon_{1}\right) r_{2} \beta \lambda_{h}}{\delta_{3}\left(\delta_{4}+\theta\right)\left(\mu_{1} \delta_{1}+\beta \lambda_{h}\right)}\right)} .
\end{aligned}
$$

Given $\varepsilon_{1}, \varepsilon_{2} \in[0,1]$, the blood stage treatment is a perfect treatment if $\varepsilon_{1}=\varepsilon_{2}=1$ and totally ineffective if $\varepsilon_{1}=\varepsilon_{2}=0$. If the reproduction number $R_{\varepsilon}$ under treatment is less than one, then $R_{\varepsilon}<R_{0}$. There is clearance of the malaria parasites, reduction in infectious reservoirs, malaria can be greatly reduced. The treatment is useless if $R_{\varepsilon}=R_{0}$ and efficacy is equal to zero. The condition $R_{\varepsilon}<1$ is necessary to guarantee that the system is locally asymptotically stable.

Theorem 4.1. The disease free equilibrium with treatment effects of blood schizontocides exists and is locally asymptotically stable if $R_{\varepsilon}<1$ and unstable if $R_{\varepsilon}>1$.

Proof. A partial differentiation of $R_{\varepsilon}$ with respect to $\varepsilon_{1}$ and $\varepsilon_{2}$, gives

$$
\begin{aligned}
& \frac{\partial R_{\varepsilon}}{\partial \varepsilon_{1}}=-\left(\left(1-\varepsilon_{2}\right) \tau_{1}\left(1+\left(1-\varepsilon_{2}\right) \tau_{2}\right)^{1 / 2}+\frac{1}{2} \cdot \frac{\tau_{3}\left(1+\left(1-\varepsilon_{2}\right) \tau_{2}\right)^{1 / 2} \tau_{4}}{\tau_{5}\left(1+\left(1-\varepsilon_{2}\right) \tau_{2}\right)^{1 / 2}}\right)<0, \\
& \frac{\partial R_{\varepsilon}}{\partial \varepsilon_{2}}=-\left(\left(1-\varepsilon_{1}\right) \tau_{1}\left(1+\tau_{2}\right)^{1 / 2}+\frac{1}{2} \cdot \frac{\tau_{2} \tau_{6}}{\left(1+\left(1-\varepsilon_{2}\right) \tau_{2}\right)^{1 / 2}}\right)<0,
\end{aligned}
$$

where

$$
\begin{aligned}
\tau_{1} & =\frac{(1-\rho) \omega \lambda_{e} r_{2}}{2\left(\delta_{2} \mu_{3}+\left(1-\varepsilon_{1}\right) \omega \lambda_{e}\right)}, \quad \tau_{2}=\frac{4 r_{1} r_{3} \theta \kappa \sigma \phi \rho\left(\delta_{2} \mu_{3}+\left(1-\varepsilon_{1}\right) \omega \lambda_{e}\right)}{\delta_{3} \omega \lambda_{e}\left(\delta_{4}+\theta\right)(1-\rho)^{2}}, \\
\tau_{3} & =\frac{\left(1-\varepsilon_{1}\right)\left(1-\varepsilon_{2}\right)^{2} r_{3} r_{2} \theta \kappa \sigma \phi \rho \omega^{2} \lambda_{e}}{\left(\delta_{2} \mu_{3}+\left(1-\varepsilon_{1}\right) \omega \lambda_{e}\right)^{2}}, \quad \tau_{4}=\frac{4 r_{1} r_{3} \theta \kappa \sigma \phi \rho \lambda_{e}}{\delta_{3} \lambda_{e}\left(\delta_{4}+\theta\right)(1-\rho)^{2}} \\
\tau_{5} & =2\left(\delta_{2} \mu_{3}+\left(1-\varepsilon_{1}\right) \omega \lambda_{e}\right)+\left(1-\varepsilon_{1}\right)\left(1-\varepsilon_{2}\right) \omega \lambda_{e}(1-\rho)^{2} \omega^{2} r_{3} \\
\tau_{6} & =\frac{\left(1-\varepsilon_{1}\right)\left(1-\varepsilon_{2}\right)(1-\rho) \omega \lambda_{e} r_{2}}{2\left(\delta_{2} \mu_{3}+\left(1-\varepsilon_{1}\right) \omega \lambda_{e}\right)} .
\end{aligned}
$$


This means that $R_{\varepsilon}$ decreases as $\varepsilon_{1}$ and $\varepsilon_{2}$ increase. $R_{\varepsilon}<1<R_{0}$ whenever the partial derivatives $\frac{\partial R_{\varepsilon}}{\partial \varepsilon_{1}}$ and $\frac{\partial R_{\varepsilon}}{\partial \varepsilon_{2}}$ are less than zero. It is also evident that treatment is $100 \%$ effective as $\varepsilon_{1}$ and $\varepsilon_{2}$ approach 1. Therefore, a highly effective drug administered to infected individuals in the asymptomatic stage can lead to effective disease control if $\lim R_{\varepsilon} \rightarrow 0$ and $R_{\varepsilon}<1$.

\section{Numerical analysis}

The numerical simulations of disease-free, and endemic equilibrium points are performed to establish long term effects. Parameter values used in the simulations are given in Table 1.

\subsection{Simulations in absence of malaria Plasmodium}

Figure 2 shows the disease-free dynamics of uninfected hepatocytes $X(t)$ in the liver and uninfected erythrocytes $Z(t)$ in the blood.

(a): uninfected Hepatocytes at $\mathrm{E}_{0}$

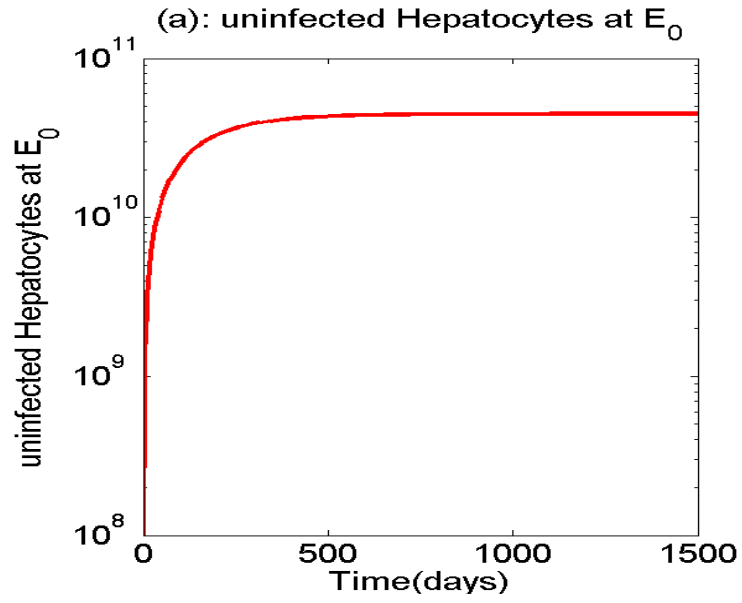

(b): uninfected Erythrocytes at $E_{0}$

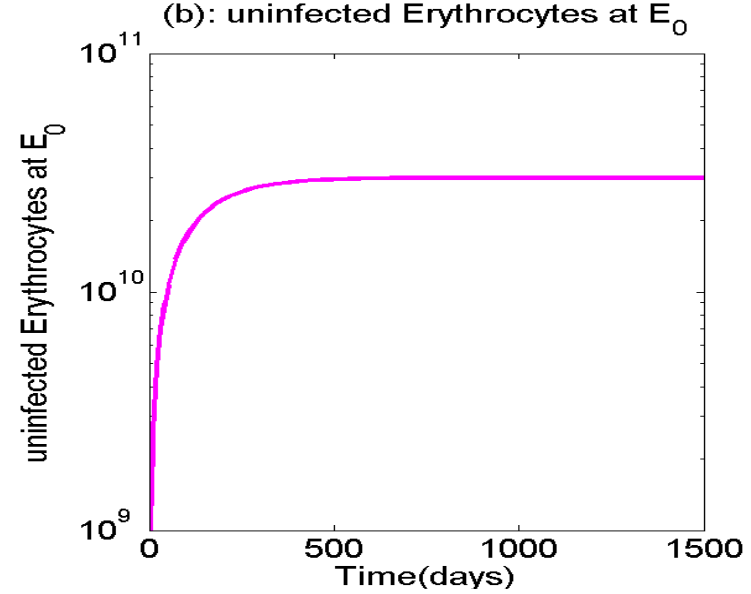

Figure 2: Simulations of disease-free equilibrium, using $\mu_{1}=0.0067$.

In Figure 2, the results do not depend on the initial values and in the absence of malaria, the susceptible hepatocytes in Figure 2 (a) and erythrocytes in Figure 2 (b) increase exponentially.

\subsection{Simulations of endemic dynamics of malaria}

The simulations show the progression of malaria Plasmodium at the three different stages.
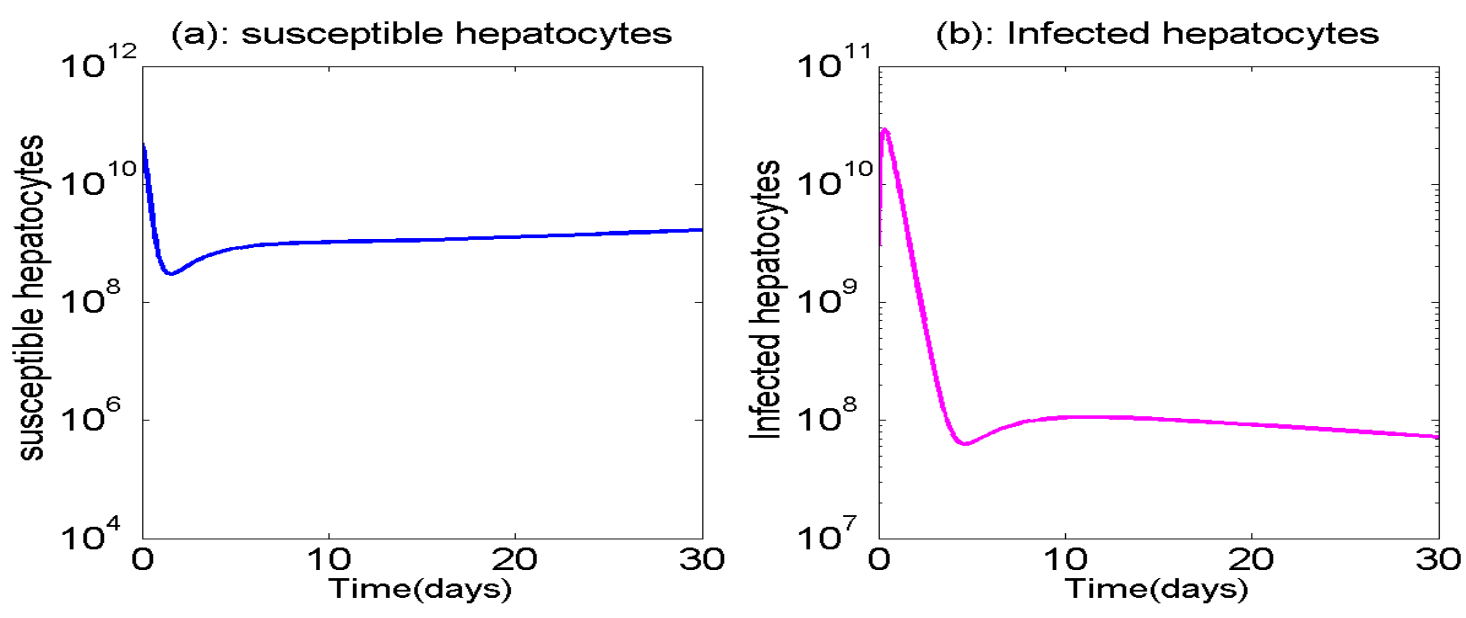

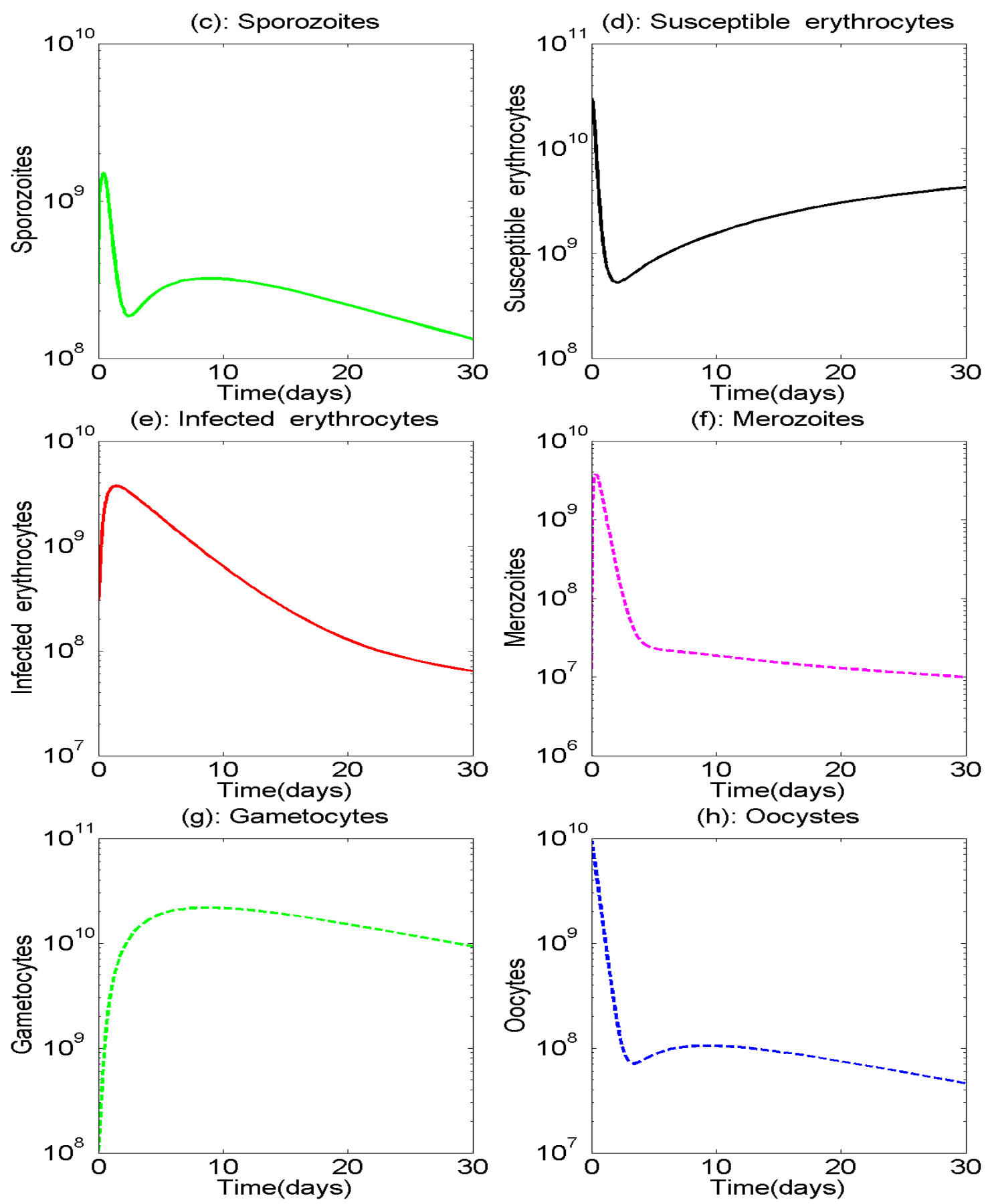

Figure 3: Simulation of the malaria Plasmodium progressions during the liver, blood and mosquito stages.

In Figure 3 (a)-(h), there is a sharp fall in the level of healthy hepatocytes in Figure 3 (a) in the liver due to infection, which is consistent with results in $[19,38,52,65,66]$. The density of sporozoites in Figure 3 (c) and infected hepatocytes in Figure 3 (b) rise. However, the density of healthy hepatocytes falls to a value below which sporozoite growth cannot be sustained. There is also a corresponding decline in sporozoites as well as infected hepatocytes. The body mechanism in an attempt to replace infected cells, trigger the recruitment of more hepatocytes. When the new recruited hepatocytes are sufficient enough for sporozoite invasion and growth, there is again a renewed slight rise in sporozoite and infected hepatocyte levels. There is also a corresponding decline in the healthy hepatocyte level. The dynamics in the liver occurs faster in the initial stages upon invasion and infection during the first 5-14 days, the period for full development of malaria parasites before they are released into the blood stream [45]. 
In Figure $3(\mathrm{~d})-(\mathrm{f})$, the dynamics of invasion and infection in the blood is faster during the first 5 days. This period captures the 48 hours life span of infected erythrocytes which concurs with Tumwiine [66]. In Figure 3 (d), erythrocytes decline sharply followed by a corresponding rise in merozoites in Figure 3 (e) and infected erythrocyte in Figure 3 (f). However, when the population density of erythrocytes declines below a level which cannot sustain parasite growth, merozoites and infected erythrocytes populations fall exponentially towards an endemic equilibrium state. Consequently, new erythrocytes are recruited by an erythropoiesis process [62] and their levels rise again. The in vivo processes in the blood take 8-10 days of serious dynamics and there after slow changes are noticed in the densities towards the stable endemic equilibrium.

The gametocyte population increases during ingestion of the erythrocytic blood meal as shown in Figure $3(\mathrm{~g})$. The gametocytes just reproduce oocysts sexually and themselves do not die in the process, thus contributing to maintenance of their high population. The gametocytes also increase because it is assumed that mosquitoes repeatedly bite the infected individual and in the process more gametocytes are recruited. The progression of the parasites eventually initiates other cycles in the susceptible population through mosquito bites. However, gametocytes decline due to natural death.

The population of the oocysts falls as shown in Figure $3(\mathrm{~h})$ because only $5 \%$ of the gametocytes are responsible for their reproduction [9]. Some factors like natural death and death during budding out of sporozoites also reduce their population. Nevertheless, the oocysts are relatively resistant to midgut digestive protease [57] and during development, they multiply in number [7] because of self-fertalisation and cross-fertalisation [61], hence a justification for the observed rise in their levels exhibited in Figure 3 (h). In the mosquito, the dynamics occurs faster in the first 6-9 days, which is in agreement with Baton [8], who observed eight days of sexual stage Plasmodium falciparum.

\subsection{The cell and parasite population dynamics for the 3 stage complete cycle}

The dynamics in each of the 3 stages and the approximated time malaria parasites take to fully develop before transmission to the next stage are shown below.
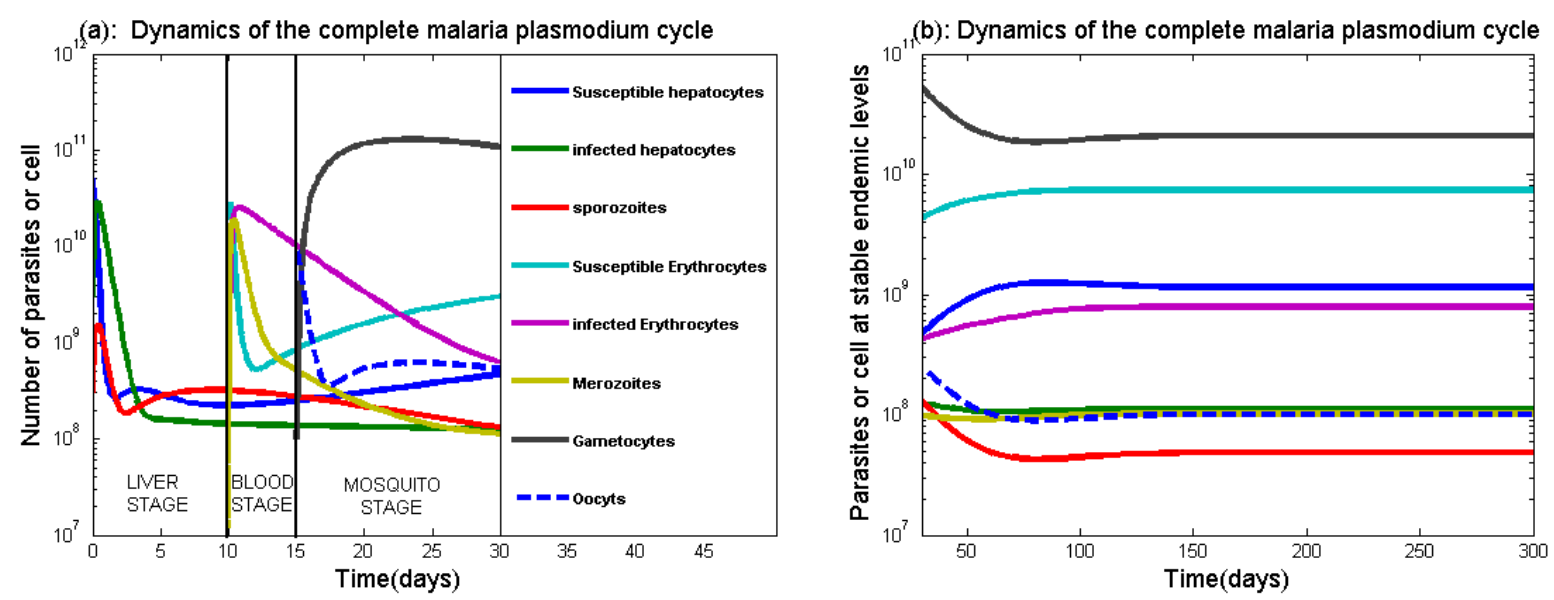

Figure 4: Simulation of the malaria Plasmodium progressions during the liver, blood and mosquito stages using parameter values in Table 1.

In Figure 4 (a), malaria parasite takes 10 to 15 days after a mosquito bite for symptoms to show up in the blood [30]. This time includes a 2 days life span of infected erythrocyte, the time merozoites take in the infected erythrocytes [65] while gametocytes take 1 to 3 days [58]. This gives an average of 10 days the parasite spends in the liver. The dynamics in the blood is considered for 5 days and about 14 days of parasite dynamics including development within the mosquito [8]. The explanation of the trends of these curves are the same as those explained in Figure 3 (a)-(h). The endemic equilibrium point (EEP) is an indication that the individual may live with malaria parasites in equilibrium with erythrocytes and 
hepatocytes. In Figure 4 (b), the EEP is attained after a period of about 150 days and it is consistent with the observations of Nannyonga [52]. In Figure 4 (b) between 50 and 100 days, malaria dynamical processes slow down and there is minimal significant changes in the levels of various parasites and cells as they tend to the steady state. Moreover it is assumed that there is always new infected mosquitoes that repeatedly bite the individual to continue the cycle to naive individuals. The body mechanisms maintain a low-grade and a generally asymptomatic parasitemia [20] and provide protection against new infections.

The protection is evidence of a lower risk of clinical disease, as indicated by the absence of illness (fever) in the presence of malaria parasites due to their lower densities that cause no malaria morbidity. In this period which starts from 50 to 100 days after infection, majority of infected adults in an endemic area rarely experience overt disease. They go about their daily routines of school, work, and household chores feeling essentially healthy despite a population of parasites in their blood that would almost universally prove lethal to a malaria-naive visitor [20] and this would cause morbidity to such a visitor. Therefore, it can be noted that an individual living in a malaria endemic area stays at the equilibrium point with different proportions of the steady states of various parasites and cells without causing morbidity.

5.4. Simulations for treatment effects in the blood

(a) Susceptible erythrocytes under treatment
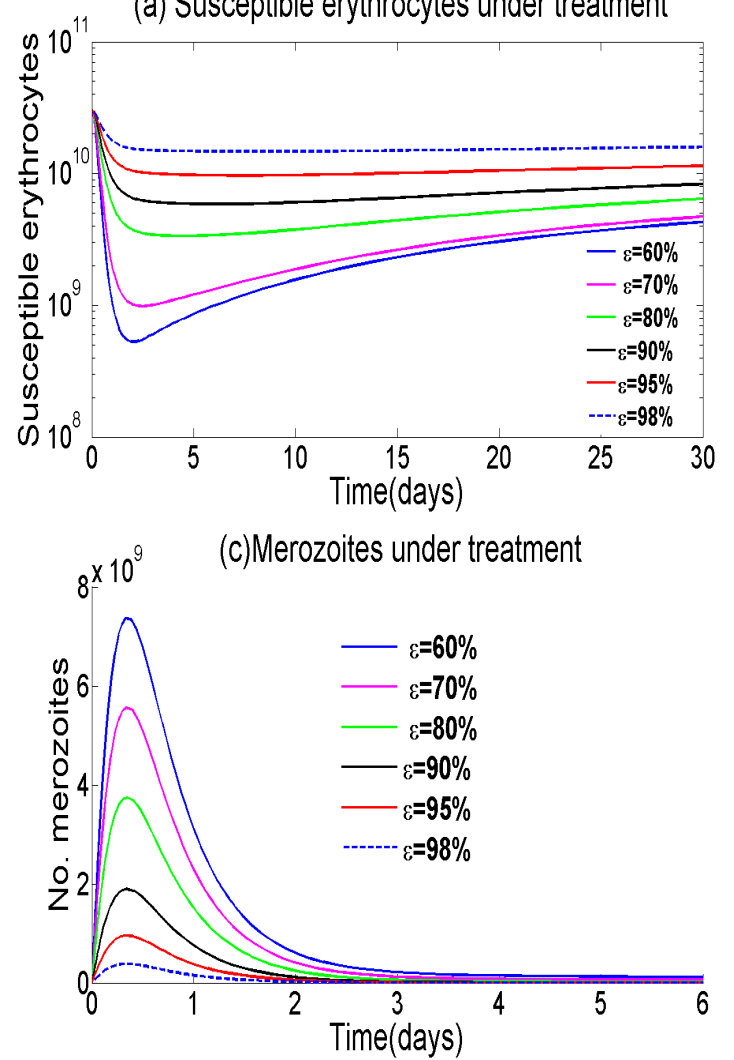

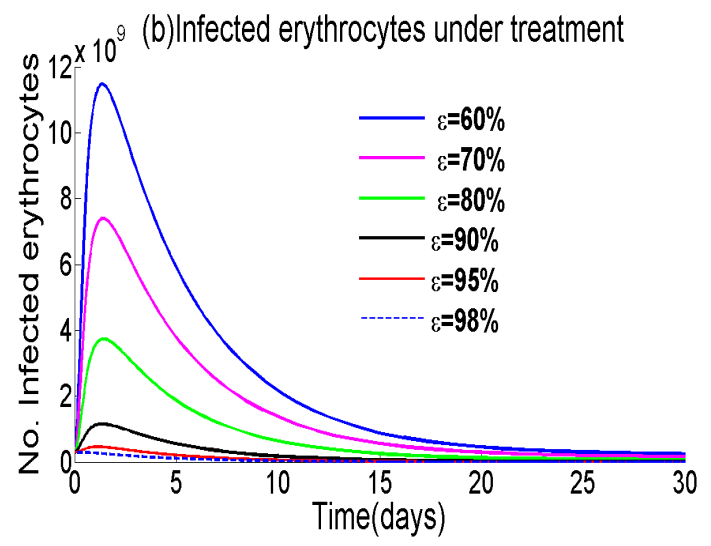

(d): Varying Reproduction numbers with treatment

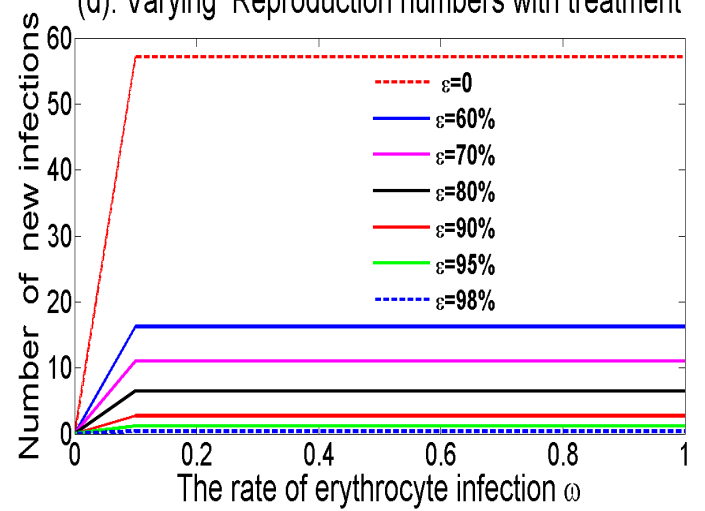

Figure 5: Simulations for treatment effects in the blood in presence of drugs with efficacies lying between $60 \%$ and $100 \%$.

In the presence of blood drugs, the number of healthy erythrocytes shown in Figure 5 (a) increases with an increase in drug effectiveness to an approximate value corresponding to the disease-free state. The population of infected erythrocytes in Figure 5 (b) declines to lower values when drugs of high efficacy are used. However, different drugs of different efficacies give almost the same benefit after 20 days of treatment. In Figure 5 (c), the highly effective drugs clear the parasites faster than those of low efficacy. After 2 to 3 days, the same treatment benefits are realized. The average number of new infections shown in Figure 5 (d), increases when the rate of interaction $\omega$ between the merozoites and erythrocytes increases. When $R_{0}>1$, the endemicity increases to a stable endemic equilibrium [15] if 
there are no more new infections. In the presence of treatment with different efficacies, $R_{0}$ decreases to very low values less than one especially whenever drug efficacies are increased to values more than $90 \%$. In Figure $5(\mathrm{~d})$, the maximum infection rate reached is 0.1 beyond which no more infection is noticed. In the presence of highly effective drugs (above 0.9), the model exhibits a forward bifurcation whenever $\mathrm{R}_{0}<1$. Hence equilibrium point reached is a disease-free equilibrium point. Therefore, the disease can be greatly reduced and can disappear from the populations.

\section{Discussion, conclusion and recommendation}

\subsection{Discussion}

A proposed mathematical model of within host dynamics of malaria life cycle has been studied. The formulated model was a modification of a study by Anderson [3]. The modification included connection of exo-erythrocyte cycle in liver, erythrocyte cycle in blood and sporogonic cycle within the mosquito. The study also incorporated the effects of treatment with the main focus on the blood stage. The positivity and existence of the system's solutions have been presented and the analysis of the model has showed that solutions exist. In the absence of treatment parameters, the model yielded a within-host reproduction number $R_{0}$ from which the most effective control strategies in assessing treatment effects in the blood were deduced.

The results in this study show that the disease-free equilibrium state is locally and globally stable whenever $R_{0}<1$ and unstable whenever $R_{0}>1$. These results are in agreement with those in $[44,52,65,66]$. The local stability was determined using eigenvalue analysis and the Routh-Hurwitz stability criterion. The approach by Castillo-Chavez [14] was used to guarantee the global stability of DFE. The center manifold theory [15] was used and the endemic equilibrium was found to be locally asymptotically stable. It was noted that some of the sensitive parameters causing the onset of infection were $\omega, \lambda_{e}, r_{1}, r_{2}, r_{3}, \sigma, \rho, \phi, k$ and $\theta$. However, the number of merozoites released into the blood from infected hepatocytes of the liver $r_{1}$, the burst size $r_{2}$ of infected erythrocytes in the blood, and the rate of infection $\omega$ of erythrocytes by the merozoites were more sensitive, and this was in line with the findings of Kamangira [44]. In this study $r_{1}$ and $k$ were significant parameters in the successful invasion and persistence of malaria. The sensitivity of these parameters leads to the progression of the disease which debilitates the the host's immunity [42]. The progression can cause death if no treatment strategy is involved.

The simulations of the model without treatment showed that infected hepatocytes, sporozoites, infected erythrocytes, merozoites and gametocytes first increase and decline towards a steady endemic state. The healthy hepatocytes, and healthy erythrocytes reduce and then increase towards a steady endemic state. The simulations with treatment showed that the number of susceptible erythrocytes increased whenever the rate of effectiveness increased. The number of infected erythrocytes and merozoites reduced whenever the rate of effectiveness increased.

The treatment strategy with high rate of drug efficacy helped to reduce the reproduction number to a value less than unity and this concurred with the findings of Kamangira [44]. Therefore, it is better to suppress the merozoites population which serves as a way of eradicating the disease at blood stage. These results are in agreement with those in $[3,23,28,34,35,44]$ who accessed the impact of chemotherapy and biological control measures on the parasite growth focussing on the erythrocytic cycle of malaria. It is suggested that the effectiveness of chemotherapy and biological measures inversely related to the lifeexpectancy of the targeted stage in the parasite's development cycle.

\subsection{Conclusions}

The model adds more details to the complex malaria life cycle and highlights the interaction features in within-host malaria infection. It is therefore necessary to combine all the stages of malaria Plasmodium in order eradicate malaria. The model is biologically realistic and can be used to check the transmission dynamics of malaria and the effects of treatment. 
However, there were challenges which were experienced in the course of carrying out this research. Due to insufficient data and literature, some of the parameters were estimated. These estimates in some cases did not portray the reality of the malaria dynamics. Hence understanding parasite biology in the liver and within the mosquito is highly desirable.

\subsection{Recommendations}

According to Millennium Development Goals (4) and (6) which are to combat HIV/AIDs, malaria and to reduce child mortality [10], it is imperative that the following recommendations be considered in order to combat malaria:

(i) There is need for further research to enable better understanding of parasite biology. Therefore adequate investment in basic biological and clinical research investigations of the malaria Plasmodium life cycle especially detecting the parasites at the liver stage is recommended. This would help to provide knowledge needed for identification of new targets, strategies, and development of successful new drugs to combat the increasing burden of malaria especially in the initial stages in the liver.

(ii) To improve on the existing treatment against blood stage malaria Plasmodium, a malaria therapy with high efficacy drugs should be maintained.

\section{Acknowledgment}

Special thanks go to the Belgian Government through the Belgium Technical Co-operation (BTC) for the financial support in part offered in this research exercise. The authors also thank Sida Phase-IV Bilateral program, Project 316 for the financial support in the publication of this work.

\section{References}

[1] C. K. Ahorlu, S. K. Dunyo, E. A. Afari, K. A. Koram, F. K. Nkrumah, Malariarelated beliefs and behaviour in Southern Ghana: Implications for treatment, prevention and control, Tropical Med. Int. Health, 2 (1997), 488-499. 1, 2.7

[2] R. M. Anderson, R. M. May, Infectious diseases of humans, Oxford Univ. Press, Oxford, (1991). 1, 1

[3] R. M. Anderson, R. M. May, S. Gupta, Nonlinear phenomena in host-parasite interactions, Parasitology, 99 (1989), 59-79. 1, 1, 6.1

[4] D. J. Austin, N. J. White, R. M. Anderson, The dynamics of drug action on the within-host population growth of infectious agents: melding pharmacokinetics with pathogen population dynamics, J. Theoret. Biol., 194 (1998), 313-339. 1, 1

[5] K. Baer, C. Klotz, S. H. Kappe, T. Schnieder, U. Frevert, Release of hepatic Plasmodium yoelii merozoites into the pulmonary microvasculature, PLoS Pathog., 3 (2007), 170-177. 1

[6] L. Bannister, G. Mitchell, The ins, outs and roundabouts of malaria, Trends Parasitol., 19 (2003), 209-213. 1

[7] L. A. Baton, L. C. Ranford-Cartwright, How do malaria ookinetes cross the mosquito midgut wall?, Trends Parasitol., 21 (2005), 22-28. 1, 5.2

[8] L. A. Baton, L. C. Ranford-Cartwright, Spreading the seeds of million-murdering death: metamorphoses of malaria in the mosquito, Trends Parasitol., 21 (2005), 573-580. 5.2, 5.3

[9] J. C. Beier, Malaria parasite development in mosquitoes, Annu. Rev. Entomol., 43 (1998), 519-543. 5.2

[10] R. Black, H. White, Targeting development: critical perspectives on the millennium development goals, Routledge Studies in Development Economics, Routledge, (2004). 1, 6.3

[11] M. R. Briones, M. Tsuji, V. Nussenzweig, The large difference in infectivity for mice of Plasmodium berghei and Plasmodium yoelii sporozoites cannot be correlated with their ability to enter into hepatocytes, Mol. Biochem. Parasitol., 77 (1996), 7-17. 1

[12] I. N. Brown, Immunological aspects of malaria infection, Adv. Immunol., 11 (1969), 267-349. 1

[13] O. Bruña-Romero, J. C. Hafalla, G. González-Aseguinolaza, G. I. Sano, M. Tsuji, F. Zavala, Detection of malaria liver-stages in mice infected through the bite of a single Anopheles mosquito using a highly sensitive real-time PCR, Int. J. Parasitol., 31 (2001), 1499-1502. 1

[14] C. Castillo-Chavez, Z. Feng, W. Huang, On the computation of $\mathrm{R}_{0}$ and its role on global stability, Mathematical approaches for emerging and reemerging infectious diseases: an introduction, Minneapolis, MN, (1999), 229-250, IMA Vol. Math. Appl., Springer, New York, (2002). 2.8, 2.8, 6.1

[15] C. Castillo-Chavez, B. Song, Dynamical models of tuberculosis and their applications, Math. Biosci. Eng., 1 (2004), 361-404. 5.4, 6.1 
[16] C. Chiyaka, W. Garira, S. Dube, Using mathematics to understand malaria infection during erythrocytic stages, Zimbabwe J. Sci. Technol., 5 (2010), 1-11 1

[17] A. F. Cowman, B. S. Crabb, Invasion of red blood cells by malaria parasites, Cell, 124 (2006), 755-766. 1

[18] F. E. Cox, History of the discovery of the malaria parasites and their vectors, Parasit. Vectors, 3 (2010), 4-8. 1

[19] A. M. Dondorp, P. A. Kager, J. Vreeken, N. J. White, Abnormal blood flow and red blood cell deformability in severe malaria, Parasitol. Today, 16 (2000), 228-232. 1, 1, 5.2

[20] D. L. Doolan, C. Dobaño, J. K. Baird, Acquired immunity to malaria, Clin. Microbiol. Rev., 22 (2009), 13-36. 5.3

[21] P. Van den Driessche, J. Watmough, Reproduction numbers and sub-threshold endemic equilibria for compartmental models of disease transmission, Math. Biosci., 180 (2002), 29-48. 2.6

[22] L. Esteva, A. B. Gumel, C. V. De LeóN, Qualitative study of transmission dynamics of drug-resistant malaria, Math. Comput. Modelling, 50 (2009), 611-630. 1

[23] J. A. Filipe, E. M. Riley, C. J. Drakeley, C. J. Sutherland, A. C. Ghani, Determination of the processes driving the acquisition of immunity to malaria using a mathematical transmission model, PLoS Comput. Biol., 3 (2007), 250-262. 6.1

[24] F. Forouzannia, A. B. Gumel, Mathematical analysis of an age-structured model for malaria transmission dynamics, Math. Biosci., 247 (2014), 80-94. 1

[25] U. Frevert, S. Engelmann, S. Zougbédé, J. Stange, B. Ng, K. Matuschewski, L. Liebes, H. Yee, Intravital observation of Plasmodium berghei sporozoite infection of the liver, PLoS Biol., 3 (2005), 189-197 1

[26] F. Frischknecht, P. Baldacci, B. Martin, C. Zimmer, S. Thiberge, J. C. Olivo-Marin, S. L. Spencer, R. Ménard, Imaging movement of malaria parasites during transmission by Anopheles mosquitoes, Cell. Microbiol., 6 (2004), 687-694. 1

[27] C. R. Garcia, R. P. Markus, L. Madeira, Tertian and quartan fevers: temporal regulation in malarial infection, J. Biol. Rhythms, 16 (2001), 436-443. 1

[28] M. Ghosh, A. A. Lashari, X.-Z. Li, Biological control of malaria: a mathematical model, Appl. Math. Comput., 219 (2013), 7923-7939. 1, 6.1

[29] S. Glushakova, D. Yin, T. Li, J. Zimmerberg, Membrane transformation during malaria parasite release from human red blood cells, Curr. Biol., 15 (2005), 1645-1650. 1

[30] M. Gomes, F. Espino, J. Abaquin C. Realon, N. Salazar, Symptomatic identification of malaria in the home and in the primary health care clinic, Bull. World Health Organ., 72 (1994), 380-389. 1, 5.3

[31] L. C. Gouagna, S. Bonnet, R. Gounoue, J. Verhave, W. Eling, R. Sauerwein, C. Boudin, Stagespecific effects of host plasma factors on the early sporogony of autologous Plasmodium falciparum isolates within Anopheles gambiae, Trop. Med. Int. Health, 9 (2004), 937-948 1

[32] B. M. Greenwood, D. A. Fidock, D. E. Kyle, S. H. Kappe, P. L. Alonso, A. Collins, Malaria: progress, perils, and prospects for eradication, J. Clin. Invest., 118 (2008), 1260-1274. 1

[33] P. J. Guerin, P. Olliaro, F. Nosten, P. Druilhe, R. Laxminarayan, F. Binka, N. J. White, Malaria: current status of control, diagnosis, treatment, and a proposed agenda for research and development, Lancet Infect. Dis., 2 (2002), 564-573. 1

[34] B. F. Hall, A. S. Fauci, Malaria control, elimination, and eradication: the role of the evolving biomedical research agenda, J. Infect. Dis., 200 (2009), 1639-1643 1, 6.1

[35] N. Hall, M. Karras, J. D. Raine, J. M. Carlton, T. W. Kooij, M. Berriman, A comprehensive survey of the Plasmodium life cycle by genomic, transcriptomic, and proteomic analyses, Science, 307 (2005), 82-86. 1, 6.1

[36] B. Hellriegel, Modelling the immune response to malaria with ecological concepts: short-term behaviour against long-term equilibrium, Proc. R. Soc. Lond. [Biol.], 250 (1992), 249-256. 1, 1

[37] H. W. Hethcote, The mathematics of infectious diseases, SIAM Rev., 42 (2000), 599-653. 1

[38] C. Hetzel, R. Anderson, The within-host cellular dynamics of bloodstage malaria: theoretical and experimental studies, Parasitol., 113 (1996), 25-38. 1, 5.2

[39] M. B. Hoshen, R. Heinrich, W. D. Stein, H. Ginsburg, Mathematical modelling of the within-host dynamics of Plasmodium falciparum, Parasitol., 121 (2000), 227-235. 1

[40] R. E. Howells, E. E. Davies, Nuclear division in the oocyst of Plasmodium berghei, Ann. Trop. Med. Parasitol., 65 (1971), 451-454 1

[41] C. J. Janse, P. F. van der Klooster, H. J. van der Kaay, M. van der Ploeg, P. J. Overdulve, DNA synthesis in Plasmodium berghei during asexual and sexual development, Mol. Biochem. Parasitol., 20 (1986), 173-182. 1

[42] C. O. Jones, H. A. Williams, The social burden of malaria: what are we measuring?, Am. J. Trop. Med. Hyg., 71 (2004), 156-161. 1, 6.1

[43] K. Kaiser, N. Camargo, S. H. Kappe, Transformation of sporozoites into early exoerythrocytic malaria parasites does not require host cells, J. Exp. Med., 197 (2003), 1045-1050. 1

[44] B. Kamangira, P. Nyamugure, G. Magombedze, A theoretical mathematical assessment of the effectiveness of coartemether in the treatment of Plasmodium falciparum malaria infection, Math. Biosci., 256 (2014), 28-41 1, 6.1

[45] S. H. Kappe, K. Kaiser, K. Matuschewski, The Plasmodium sporozoite journey: a rite of passage, Trends Parasitol., 19 (2003), 135-143 1, 5.2

[46] A. Kaushansky, P. Metzger, A. Douglass, S. Mikolajczak, V. Lakshmanan, H. S. Kain, S. H. Kappe, Malaria parasite liver stages render host hepatocytes susceptible to mitochondria-initiated apoptosis, Cell Death Dis., 4 (2013), 9 pages. 1

[47] D. K. Kochar, A. Das, S. K. Kochar, V. Saxena, P. Sirohi, S. Garg, Severe Plasmodium vivax malaria: a report on serial cases from Bikaner in northwestern India, Am. J. Trop. Med. Hyg., 80 (2009), 194-198. 1 
[48] M. Kung'aro, L. S. Luboobi, F. Shahada, Reproduction number for yellow fever dynamics between primates and human beings, Commun. Math. Biol. Neurosci., 2014 (2014), 24 pages. 3

[49] G. Magombedze, P. Nduru, C. P. Bhunu, S. Mushayabasa, Mathematical modelling of immune regulation of type 1 diabetes, Biosyst., 102 (2010), 88-98. 2.4

[50] G. K. Michalopoulos, M. C. DeFrances, Liver regeneration, Science, 276 (1997), 60-66.

[51] M. M. Mota, G. Pradel, J. P. Vanderberg, J. C. Hafalla, U. Frevert, R. S. Nussenzweig, V. Nussenzweig, A. Rodríguez, Migration of Plasmodium sporozoites through cells before infection, Science, 291 (2001), 141-144. 1, 1

[52] B. Nannyonga, G. Mwanga, H. Haario, I. Mbalawata, M. Heilio, Determining parameter distribution in within-host severe P. falciparum malaria, Biosyst., 126 (2014), 76-84. 1, 5.2, 5.3, 6.1

[53] O. Oddoux, A. Debourgogne, A. Kantele, C. Kocken, T. S. Jokiranta, Identification of the five human Plasmodium species including P. knowlesi by real-time polymerase chain reaction, Eur. J. Clin. Microbiol. Infect. Dis., 30 (2011), 597-601 1

[54] W. Peters, Antimalarial drugs and their actions, Postgrad. Med. J., 49 (1973), 573-583. 1

[55] D. J. Rodriguez, L. Torres-Sorando, Models of infectious diseases in spatially heterogeneous environments, Bull. Math. Biol., 63 (2001), 547-571. 1

[56] R. Ross, Some a priori pathometric equations, BMJ, 1 (1915), 540-564.

[57] R. E. Sinden, The cell biology of sexual development in Plasmodium, Parasitol., 86 (1983), 7-28. 1, 5.2

[58] R. E. Sinden, A proteomic analysis of malaria biology: integration of old literature and new technologies, Int. J. Parasitol., 34 (2004), 1441-1450. 1, 5.3

[59] T. G. Smith, D. Walliker, L. C. Ranford-Cartwright, Sexual differentiation and sex determination in the Apicomplexa, Trends Parasitol., 18 (2002), 315-323. 1

[60] G. Snounou, S. Viriyakosol, W. Jarra, S. Thaithong, K. N. Brown, Identification of the four human malaria parasite species in field samples by the polymerase chain reaction and detection of a high prevalence of mixed infections, Mol. Biochem. Parasitol., 58 (1993), 283-292. 1

[61] A. M. Talman, O. Domarle, F. E. McKenzie, F. Ariey, V. Robert, Gametocytogenesis: the puberty of Plasmodium falciparum, Malar. J., 3 (2004), 24 pages. 1, 5.2

[62] J. J. Thibodeaux, Modeling erythropoiesis subject to malaria infection, Math. Biosci., 225 (2010), 59-67. 5.2

[63] M. Torii, K. I. Nakamura, K. P. Sieber, L. H. Miller, M. Aikawa, Penetration of the mosquito (aedes aegypti) midgut wall by the ookinetes of Plasmodium gallinaceum, J. Protozool., 39 (1992), 449-454. 1

[64] A. Trimnell, A. Takagi, M. Gupta, T. L. Richie, S. H. Kappe, R. Wang, Genetically attenuated parasite vaccines induce contact-dependent CD8 $+T$ cell killing of Plasmodium yoelii liver stage-infected hepatocytes, J. Immunol., 183 (2009), 5870-5878. 1

[65] J. Tumwiine, S. Luckhaus, J. Y. T. Mugisha, L. S. Luboobi, An age-structured mathematical model for the within host dynamics of malaria and the immune system, J. Math. Model. Algorithms, 7 (2008), 79-97. 1, 5.2, 5.3, 6.1

[66] J. Tumwiine, J. Y. T. Mugisha, L. S. Luboobi, A mathematical model for the dynamics of malaria in a human host and mosquito vector with temporary immunity, Appl. Math. Comput., 189 (2007), 1953-1965. 5.2, 6.1

[67] T. N. Wells, J. N. Burrows, J. K. Baird, Targeting the hypnozoite reservoir of Plasmodium vivax: the hidden obstacle to malaria elimination, Trends Parasitol., 26 (2010), 145-151. 1

[68] N. J. White, D. Chapman, G. Watt, The effects of multiplication and synchronicity on the vascular distribution of parasites in falciparum malaria, Trans. R. Soc. Trop. Med. Hyg., 86 (1992), 590-597. 1

[69] World Health Organization, Guidelines for the treatment of malaria, World Health Organization, Geneva, Switersland, (2006). 1

[70] World Health Organization, World malaria report 2012, World Health Organization, Geneva, (2012). 1 